\title{
Proliferative activity in bilateral paraneoplastic melanocytic proliferation and bilateral uveal melanoma
}

\author{
C M Mooy, P T V M de Jong, C Strous
}

Bilateral diffuse uveal melanocytic hyperplasia (BDUMH) is a rare but well known paraneoplastic syndrome, ${ }^{1}$ associated with systemic malignancy. An essential feature of this syndrome is a preponderantly benign looking cytology of the melanocytic tumours. ${ }^{2}$ We describe the clinical and histopathological features of BDUMH with active proliferation and bilateral ciliary body malignant melanomas. The multiple mucosal and skin pigmentations are an unusual feature of this syndrome.

\section{Case report}

In 1990, an 82-year-old man presented with painless, bilateral visual loss for 3 weeks. He had been known to have a low grade non-Hodgkin's lymphoma for 6 years, which was in remission. Over 1 year he developed multiple pigmentations on his penis, the oral mucosa, and the skin, which were diagnosed as lentigo simplex.

Ophthalmic clinical evaluation revealed visual acuities of 0.7 in both eyes. Fundus examination revealed a heavily pigmented tumour in the posterior pole of both eyes (Fig 1). The peripheral part of both eyes contained multiple sharply defined, heavily pigmented areas. The clinical differential diagnosis included Gardner's syndrome and paraneoplastic syndrome.

In 1991 a bronchus carcinoma was detected, from which the patient died in 1992. Autopsy was not permitted; however, permission was given to remove the eyes.

On microscopic examination the choroid was diffusely infiltrated by plump spindle cells and

\author{
Erasmus University \\ Rotterdam, PO Box 1738 , \\ 3000 DR Rotterdam, The \\ Netherlands \\ Department of Pathology \\ and Ophthalmology \\ C M Mooy \\ Department of \\ Ophthalmology \\ P T V M de Jong \\ Streekziekenhuis \\ Walcheren \\ C Strous \\ Correspondence to: \\ C M Mooy, MD. \\ Accepted for publication \\ 10 January 1994
}

Figure 1 Fundus of the right eye, with a heavily pigmented tumour in the posterior pole, which on histological examination was consistent with melanocytoma. small polyhedral cells. A few inconspicuous nucleoli were observed. Bleached sections of the heavily pigmented nodules showed a necrotic centre surrounded by large polyhedral cells with a basophilic central nucleus, compatible with melanocytoma cells. In both eyes the ciliary body was circumferentially thickened by atypical polyhedral cells with large nuclei, an irregular chromatin pattern and prominent nucleoli. A mitosis was sporadically observed. The tumour cells infiltrated the iris basis, the chamber angle, the trabecular system, and the pigment epithelium. Extrascleral, subconjunctival growth was noted in the right eye. In the macula focal depigmentations of the retinal pigment epithelium was observed, with atrophy of the photoreceptors.

On immunohistochemical examination the uveal melanocytes and the malignant melanoma cells stained strongly positive with HMB-45, S-100, and NKI-C3. There was weak staining with enolase and negative staining with Leu-7 and neurofilaments. Uveal melanocytes showed at different places positive nuclear staining with a proliferation associated nuclear antigen (Ki-67) (Fig 2). The positive staining was observed in less than $1 \%$ of the cells.

On flow cytometric analysis, both the benign melanocytic hyperplasia and the ciliary body melanoma were diploid.

\section{Comment}

Paraneoplastic BDUMH is a rare, but well known paraneoplastic syndrome associated with systemic malignancy. ${ }^{1}$ The primary tumour, when identified, has been found in varying organs. Our patient was known to have nonHodgkin's lymphoma, which was in remission at the time of visual loss. In most patients described with BDUMH the visual complaints preceded the symptoms related to the primary tumour. The ocular and extraocular pigmentations in our patient developed before the clinical detection of the bronchus carcinoma.

Although a preponderantly benign looking cytology of the melanocytic hyperplasia has been mentioned as an essential feature of this syndrome, bilateral multiple uveal melanomas occurred in four patients described. ${ }^{34}$

The strong expression of HMB-45 in the melanocytic hyperplasia of our patient is consistent with melanocytic activation. ${ }^{56}$ Proliferation of the uveal melanocytes was focally demon- 


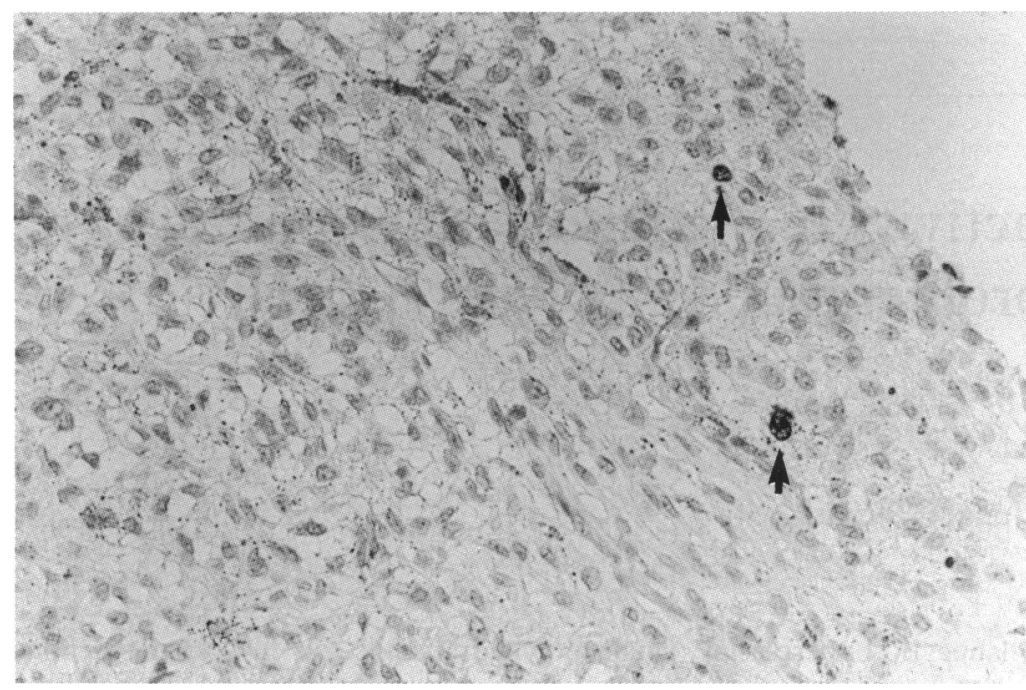

Figure 2 Photomicrograph of positive nuclear staining with Ki-67 $(\times 300)$.

strated by a proliferation associated antigen. Both the benign melanocytic hyperplasia and the melanoma were diploid, which is consistent with relatively benign tumours. Active proliferation may have led to malignant degeneration. The stimulus which leads to active proliferation of pre-existing anomalous melanocytes (that is, congenital uveal melanocytosis), is probably induced by a tumoral growth factor secreted by the primary tumour. ${ }^{1}$ The visual loss in our patient can be explained by the depigmentation of the retinal pigment epithelium and the photoreceptor atrophy.

The combination of Ota/Ito's naevus and BDUMH has been described ${ }^{3}$; however, multiple extraocular lentiginous pigmentations have so far not been recognised as part of this rare paraneoplastic syndrome.

Presented at the 32nd meeting of the European Ophthalmic Pathology Society, Sitges, 17-20 May, 1993.

1 Borruat FX, Othenon-Girard P, Uffer S, Othenin-Girard B Regli F, Hurlimann J. Natural history of diffuse uvea melanocytic proliferation. Case report. Ophthalmology 1992 ; 99: 1698-704.

2 Barr CC, Zimmerman LE, Curtin VT, Font RL. Bilateral diffuse melanocytic uveal tumors associated with systemic
malignant neoplasms. Arch Ophthalmol 1982; 100: 249-55.

3 Prause JU, Jensen OA, Eisgart F, Hansen U, Kieffer M. Bilateral diffuse malignant melanoma of the uvea associated with large cell carcinoma, giant cell type, of the lung. Case report of a newly described syndrome. Ophthalmologica 1984 189: 221-8.

4 Margo CE, Pavan PR, Gendelman D, Gragoudas E. Bilatera melanocytic uveal tumors associated with systemic malignancy: malignant melanomas or benign paraneoplastic syndrome? Retina 1987; 7: 137-41.

5 Bournier MN, McLean IW, Gamel JW. Immunohistochemica evaluation of uveal melanocytic tumors. Expression of HMB-45, S-100 protein, and neuron-specific enolase. Cancer 1991; 68: 809-14.

6 Steuhl KP, Rohrbach JM, Knorr M, Thiel HJ. Significance, specificity, and ultrastructural localization of $\mathrm{HMB}-45$ antispecificity, and ultrastructural localization of HMB-45 anti208-15.

\title{
Adenoma of the pigmented ciliary epithelium
}

\author{
Ian G Rennie, Michael K Faulkner, M Andrew Parsons
}

\section{University of Sheffield, Department of Ophthalmology and Orthoptics \\ I G Rennie}

Department of Pathology M K Faulkner

\section{The Ophthalmic}

Sciences Unit

M A Parsons

Correspondence to: Mr I G Rennie, Department of Ophthalmology and Orthoptics, Royal

Hallamshire Hospital, Glossop Road, Sheffield S10 2JF.

Accepted for publication 13 December 1993
Tumours of the ciliary body pigment epithelium are extremely uncommon..$^{1-5}$ The correct diagnosis is rarely made clinically and, almost invariably, the eye is enucleated or the lesion resected because a malignant melanoma of the ciliary body is suspected. We describe a locally invasive tumour arising from the pigment epithelium of the ciliary body which was removed by surgical resection.

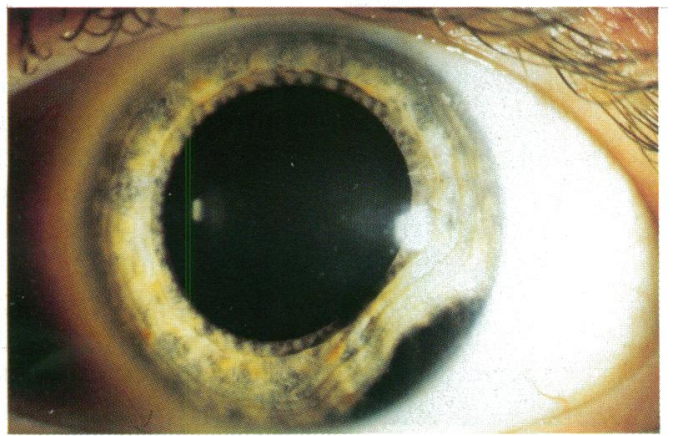

Figure 1 Clinical appearance of the tumour. Note the local distortion of the iris and the multinodular surface of the lesion.

\section{Case report}

A 40-year-old man was referred to this unit with a pigmented tumour arising from the ciliary body of his left eye. The patient had noted a black mark on the periphery of his left iris 1 month before this which had prompted his referral. On examination his uncorrected visual acuities were $6 / 5$ in both eyes. A deeply pigmented multinodular mass was noted in the inferotemporal iridocorneal angle of the left eye (Fig 1). The lesion, which occupied approximately two clock hours, had displaced the adjacent iris anteriorly causing a focal shallowing of the anterior chamber. Fundurscopy revealed a displacement of the ciliary processes overlying the lesion. Transillumination through a dilated pupil revealed an area of shadowing corresponding to this area. The intraocular pressures were $23 \mathrm{~mm} \mathrm{Hg}$ in both eyes. The remainder of the ocular examination was unremarkable. A full physical examination, including liver enzyme profile, chest $x$ ray, and abdominal ultrasound was normal. Clinically, the lesion was thought to be a malignant melanoma arising from the ciliary body with iridocorneal angle invasion. The tumour was 